\title{
Tabela de Esperança de Vida de Trichogramma pretiosum Riley em Ovos de Trichoplusia ni Hübner em Diferentes Condicionamentos Térmicos
}

\author{
José Romário de Carvalho1 ${ }^{\bowtie}$, Dirceu Pratissoli¹, Iana Pedro da Silva Quadros², João Paulo Pereira Paes ${ }^{1}$, Ingrid \\ Schimidt Kaiser ${ }^{1}$, Hígor de Souza Rodrigues ${ }^{1}$, Amanda Carlos Túller1 \& Vitor Zuim ${ }^{1}$
}

1. Universidade Federal Espírito Santo, Departamento de Produção Vegetal, Programa de Pós-graduação em Produção Vegetal, e-mail: jromario carvalho@hotmail.com (Autor para correspondência ${ }^{\bowtie}$ ), dirceu.pratissoli@gmail.com, joaopauloppaes@hotmail.com, ingrid_schimidt@hotmail.com, higorsr@live.com, amandatuller@hotmail.com, vitorzuim_es@hotmail.com. 2. Universidade Federal do Espírito Santo, Departamento de Biologia, e-mail: ianaquadros@hotmail.com.

\section{EntomoBrasilis 5 (3): 173-178 (2012)}

Resumo. O objetivo deste trabalho foi estudar a influência da temperatura sobre parametros demográficos do parasitóide de ovos, Trichogramma pretiosum Riley (Hymenoptera: Trichogrammatidae), criados em ovos da lagarta falsa-medidera-das-crucíferas, Trichoplusia ni Hübner (Lepidoptera: Noctuidae), em condições de laboratório. Para tal foram confeccionadas cartelas azuis celeste contendo ovos de T. ni que foram oferecidos ao parasitismo de $T$. pretiosum por 24 horas nas temperaturas de 18, 21, 24, 27, 30 e $33^{\circ} \mathrm{C}$. Após esse período, os parasitóides foram retirados e as cartelas mantidas nos mesmos condicionamentos térmicos até a emergência dos descendentes. A partir da emergência dos descendentes, fêmeas de T. pretiosum foram individualizadas em tubos tipo Eppendorf sendo alimentadas com mel e mantidas nas temperaturas de origem. A duração do período ovo-adulto e a longevidade das fêmeas foram fortemente influenciadas pelo regime térmico estudado, sendo inversos ao seu aumento (16,5 a 4,5 dias, para o período ovo-adulto; e 11,9 a 5,5 dias, para longevidade das fêmeas). O percentual de emergência dos descendentes, número de descendentes por ovo e a razão de sexos não sofreram influencia estatisticamente significativa. A sobrevivência $\left(L_{x}\right)$ de $T$. pretiosum foi mais influenciada nas temperaturas 27,30 e $33^{\circ} \mathrm{C}$. A esperança de vida dos adultos $\left(e_{\gamma}\right)$ foi maior nas temperaturas inferiores $\left(18,21 \mathrm{e} 24^{\circ} \mathrm{C}\right)$ verificando-se, em média, expectativas de vida de 32 , 28 e 26 dias, respectivamente. Desta maneira verificou-se que a temperatura pode ser o fator limitante para o desenvolvimento e sobrevivência de $T$. pretiosum em programas de controle biológico de $T$. ni.

Palavras-chave: Insecta; Parasitoide de ovos; Tabela de vida; Temperatura.

\section{Table of Life Expectancy of Trichogramma pretiosum Riley in Eggs of Trichoplusia ni Hübner Different Thermal Conditioning}

Abstract. The objective of this work was to study the influence of temperature on demographic parameters of the egg parasitoid, Trichogramma pretiosum Riley (Hymenoptera: Trichogrammatidae) reared on eggs of cabbage looper, Trichoplusia ni Hübner (Lepidoptera: Noctuidae) in conditions the laboratory. To this end, we made sky blue cards containing eggs of $T$. ni that were offered to the parasitism of $T$. pretiosum for 24 hours at temperatures of $18,21,24,27,30$ and $33^{\circ} \mathrm{C}$. After this period, the parasitoids were removed and kept the cards in the same thermal constraints to the emergence of offspring. From the emergence of offspring, females of T. pretiosum were individually placed in Eppendorf tubes being fed honey and kept at temperatures of origin. The duration of egg-adult period and female longevity were strongly influenced by thermal regime, being opposite to its increase (16.5 to 4.5 days, for the duration of egg-adult period; and 11.9 to 5.5 days, for and female longevity). The percentage of emergence of offspring, number of offspring per egg and sex ratio were not influenced statistically significant. The survival $\left(L_{x}\right)$ of $T$. pretiosum was more influenced at temperatures 27,30 and $33^{\circ} \mathrm{C}$. Life expectancy of adults $(e)$ was higher in lower temperatures $\left(18,21\right.$ and $\left.24^{\circ} \mathrm{C}\right)$, being verified, on average, life expectancy of 32, 28 and 26 days, respectively. Thus it was found that the temperature can be the limiting factor for the development and survival of T. pretiosum in biological control programs of $T$. ni.

Keywords: Egg parasitoid; Insecta; Life table; Temperature.

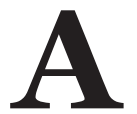

lagarta mede-palmo Trichoplusia ni Hübner (Lepidoptera: Noctuidae) é uma praga cosmopolita que apresenta uma gama de hospedeiros. Dentre os quais, destacam-se várias culturas de importância econômica como: brássicas, solanáceas, cucurbitáceas, algodão, soja, além de plantas daninhas (JANMAAT \& Myers 2003; Milanez et al. 2009). Em algumas regiões dos Estados Unidos e Canadá, $T$. ni é considerada a principal praga das brássicas, acarretando sérios problemas para as culturas tanto em campo como em casas de vegetação, destacando-se frente outras pragas de grande importância como Plutella xylostella L. (Lepidoptera: Plutellidae) e Pieris rapae L. (Lepidoptera: Pieridae) (Burkness \& Hutchison 2009). No Brasil esse inseto tem provocado enormes prejuízos especialmente entre as hortaliças folhosas, que podem ter sua comercialização inviabilizada devido aos orifícios causados pela alimentação das lagartas.
Tradicionalmente, o controle dessa praga é realizado com aplicações de inseticidas de amplo espectro (Grecco et al. 2010). Devido ao alto custo sócio-econômico destes produtos, a busca de alternativas para controle de pragas que combinem eficiência e compatibilidade ecológica é necessária. Os agentes biológicos compõem uma ferramenta importante para a redução do uso exessivo de inseticidas (Pratissoli et al. 2008). De acordo com Godin \& Boivin (1998), estes organismos podem promover a regulação da população de insetos-praga abaixo do nível de dano econômico, fato observado com a utilização de parasitóides em agroecossistema de brássicas.

Parasitóides do gênero Trichogramma (Hymenoptera: Trichogrammatidae) destacam-se pela ampla distribuição geográfica e atuação sobre ovos de vários hospedeiros, principalmente da ordem Lepidoptera (Pratissoli et al. 2002). HASSAN (1997) e PARRA et al. (2002) ressaltam que a obtenção 
de sucesso na utilização de Trichogramma em programas de controle biológico depende de várias etapas como coleta, identificação, manutenção em laboratório, seleção de espécies e, ou linhagens do parasitóide, além de condições ambientais.

A seleção de espécies e, ou linhagens de Trichogramma é feita com base em características biológicas. Milanez et al. (2009), por sua vez, selecionaram Trichogramma pretiosum Riley linhagem Tspd, dentre outras, por ser a mais promissora para o controle de $T$. ni. Contudo, estudos devem ser conduzidos a fim de avaliar Trichogramma sobre o hospedeiro de destino, em interaçao com fatores ambientais adversos. Dentre os fatores abióticos, a temperatura tem sido relatada como a que mais afeta o desenvolvimento deste parasitóide (SAMARA et al. 2011).

As tabelas de vida permitem a compreensão da dinâmica populacional das espécies, representando um excelente método para estudos biológicos inter e intraespecíficos (PRATISSOLI \& PARRA 2000; IRANIPOUR et al. 2010). Deste modo, visando atender a diversidade de regiões produtoras de brássicas, o presente estudo teve por objetivo obter as tabelas de esperança de vida de T. pretiosum em T. ni sob diferentes temperaturas, almejando a sua implementação no manejo desta praga.

\section{MATERIAL E MÉTODOS}

O experimento foi conduzido no Setor de Entomologia do Núcleo de Desenvolvimento Científico e Tecnológico em Manejo Fitossanitário (NUDEMAFI) do Centro de Ciências Agrárias da Universidade Federal do Espírito Santo, Alegre.

Criação e manutenção de Trichoplusia ni. Pupas de $T$. ni da criação estoque do setor de Entomologia do NUDEMAFI foram sexadas e acondicionadas em potes plásticos contendo no fundo papel filtro umedecido. Ocorrendo a emergência dos adultos, estes foram transferidos para gaiola de estrutura de madeira ( $60 \times 50 \times 50 \mathrm{~cm}$ ) que possuía no seu interior uma folha de couve, acondicionada com o pecíolo em um frasco de vidro de 300 mL com água, como local de oviposição das fêmeas. Para a alimentação dos adultos foi oferecida uma solução de mel a 10\% em frascos de $20 \mathrm{~mL}$, contendo chumaço de algodão em contato com a solução, sendo esse alimento renovado a cada 48 horas. Diariamente pela manhã, a folha de couve era substituída por uma nova, e a que continha ovos era acondicionada em recipiente plástico. Após a eclosão três lagartas foram transferidas e mantidas em tubos de vidro $(8,5 \times 2,5 \mathrm{~cm})$ até a fase de pupa, sendo utilizada para sua a alimentação, a dieta artificial proposta por GREENE et al. (1976).

Criação e manutenção do Trichogramma. A criação e multiplicação foram realizadas em ovos do hospedeiro alternativo Anagasta kuehniella Zeller (Lepidoptera: Pyralidae) conforme a metodologia descrita por PARRA et al. (2002). Utilizando goma arábica $(30 \% \mathrm{p} / \mathrm{v})$, os ovos de $A$. kuehniella foram colocados em cartelas de cartolina azul celeste (8,o x 2,0 $\mathrm{cm})$ que em seguida foram expostas a lâmpada germicida para inviabilização, por um período de 45 minutos. Isso se torna necessário para evitar o canibalismo dos ovos parasitados pelas lagartas de A. kuehniella (Pratissoli et al. 2010). Em seguida, as cartelas foram acondicionadas em tubos de vidro $(8,5 \times 2,5 \mathrm{~cm}) \mathrm{e}$ fêmeas do parasitóide foram introduzidas. Por sua vez, gotículas de mel foram depositadas no interior dos tubos para servir de suplemento alimentar aos parasitóides. Esta etapa foi conduzida em câmaras climatizadas, reguladas na temperatura de $25 \pm 1^{\circ} \mathrm{C}$, UR: $70 \pm 10 \%$ e fotofase de $14 \mathrm{~h}$ (Pratissoli et al. 2010).

Tabelas de esperança de vida. Vinte cartelas de cartolina azul celeste $(2,5 \times 0,3 \mathrm{~cm})$ contendo 25 ovos foram individualizadas em tubos tipo Eppendorf $(3,0 \mathrm{~mL})$, nos quais foram introduzidas 2 fêmeas (idade o-6 horas) de T. pretiosum linhagem Tspd, e mantidos em câmaras climatizadas, reguladas a $70 \pm 10 \%$ de umidade relativa (UR), fotofase de 14 horas e temperaturas de $18,21,24,27,30,33 \pm 1^{\circ} \mathrm{C}$, provenientes das estimativas mínima, média e máxima anuais de regiões produtoras. Após 5 horas de parasitismo, as fêmeas foram retiradas com auxílio de pincel e os tubos foram mantidos nas mesmas condições. Essa metodologia utilizada por Pratissoli \& PARRA (2000) implica na obtenção de ovos do parasitóide da mesma idade. Passados três dias, 100 ovos parasitados (coloração escura), de cada temperatura, foram selecionados. As observações foram realizadas diariamente para determinação do período de desenvolvimento ovo-adulto, percentagem de emergência, número de descendentes por ovo e razão de sexos.

A partir da emergência dos adultos, 40 fêmeas (idade o-6 horas), provenientes de cada temperatura $(18 ; 21 ; 24 ; 27 ; 30$ e $33 \pm 1^{\circ} \mathrm{C}$ ), foram individualizadas em tubos tipo Eppendorf $(3,0$ $\mathrm{mL}$ ) e retornaram para os respectivos condicionamentos. Como alimento foi utilizado uma gotícula de mel, sendo substituídas a cada 48 horas. As características avaliadas foram: sobrevivência e longevidade das fêmeas.

As tabelas de vida foram calculadas baseando-se em Silveira Neto et al. (1976) e KREBS (1994). Para a elaboração da tabela de esperança de vida determinaram-se os valores de número de sobreviventes $\left(L_{x}\right)$ [para facilidade dos cálculos foi mantida a proporção de 1:1 (adulto: ovo)], número de indivíduos mortos $\left(d_{x}\right)$, estrutura etária $\left(E_{x}\right)$, numero de insetos vivos de idade $x$ além da idade $x\left(T_{x}\right)$, esperança de vida $\left(e_{x}\right)$ e probabilidade de morte na idade $x\left(100 q_{x}\right)$, onde:

$$
\begin{aligned}
& E_{x}=\left(L_{x}+L_{x+1}\right) / 2 \\
& T_{x}=E_{x}+E_{x+1}+E_{x+2}+\cdots+E_{w}, \text { onde } w \text { é o ultimo estágio. } \\
& e_{x}=T_{x} / L_{x} \\
& 100 q_{x}=\left(d_{x} / L_{x}\right) \cdot 100
\end{aligned}
$$

Análise Estatística. O delineamento utilizado foi o inteiramente casualizado sendo os tratamentos constituídos pelas seis temperaturas e os dados submetidos a analise de regressão. A escolha da equação que melhor se ajustou aos dados foi baseada no fenômeno em estudo e na significância dos coeficientes de regressão $\left(\beta_{i}\right)$ e da regressão pelo teste $F(\mathrm{P}<0,05)$ e no coeficiente de determinação $\left(R^{2}\right)$.

Para percentagem de emergência, número de descendentes por ovo e razão de sexos foi utilizado o delineamento inteiramente casualizado com seis tratamentos (temperaturas) sendo os dados submetidos a analise de variância.

\section{RESULTADOS}

A duração do período ovo-adulto e a longevidade das fêmeas de T. pretiosum linhagem Tspd em ovos de T. ni foram afetados significativamente pela temperatura $(\mathrm{F}=2342,8 ; \mathrm{gl}=2,230$; $\mathrm{P}<0,0001$ para o período ovo-adulto; e $\mathrm{F}=127,7 ; \mathrm{gl}=1,228$; $\mathrm{P}<\mathrm{O}, 005$ para a longevidade das fêmeas) sendo maior nas temperaturas mais baixas (Figura 1A e 1B).

A percentagem de emergência, o número de descendentes emergidos por ovo de $T$. ni e a razão de sexos de $T$. pretiosum linhagem Tspd não foram afetados pela temperatura $(\mathrm{P}>0,05)$, apresentando em média 96,18\%, 1,7 indivíduos/ovo e 69,35\% de fêmeas, respectivamente.

O número de sobreviventes $\left(L_{x}\right)$ do parasitóide no início da fase adulta sofreu uma queda nas temperaturas mais elevadas $(27,30$ e $33^{\circ} \mathrm{C}$ ) (Figura 2). Nas temperaturas mais baixas $\left(18\right.$ e $\left.21^{\circ} \mathrm{C}\right)$ a sobrevivência das fêmeas adultas foi maior quando comparado com a mais elevada $\left(33^{\circ} \mathrm{C}\right)$.

A mortalidade $\left(d_{x}\right)$ de adultos de $T$. pretiosum linhagem Tspd iniciou a partir de diferentes idades nas diferentes temperaturas estudadas (Figura 2). Na temperatura de $18^{\circ} \mathrm{C}$ a mortalidade 
iniciou quando os adultos já se encontravam no $8^{\circ}$ dia de idade. Para as temperaturas de 24 e $27^{\circ} \mathrm{C}$ isso ocorreu a partir do $6^{\circ}$ dia, enquanto que a 21,30 e $33^{\circ} \mathrm{C}$ as mortalidades tiveram seu inicio a partir do $5^{\circ}, 4^{\circ}$ e $1^{\circ}$ dia de idade, respectivamente. A partir dessas idades, a mortalidade dos adultos apresentou um aumento em todas as temperaturas, gerando probabilidades de morte $\left(100 q_{x}\right)$ de 53,$3 ; 41,7 ; 87,5$ e $80,0 \%$, para as temperaturas de $18,21,24$ e $27^{\circ} \mathrm{C}$, respectivamente, entre o $12^{\circ}$ e $13^{\circ}$ dias de idade. As probabilidades de morte superiores a 50\% para as temperaturas de 30 e $33^{\circ} \mathrm{C}$ ocorreram a partir do $9^{\circ}$ e $7^{\circ}$ dias de idade dos adultos, respectivamente (Figura 2).

A esperança de vida $\left(e_{x}\right)$ de $T$. pretiosum linhagem Tspd no decorrer dos dias decaiu com o aumento da idade dos parasitóides, se ajustando ao modelo linear (Figura 2). Para o primeiro dia, a $e_{x}$ apresentou um comportamento inverso ao acréscimo de temperatura, variando entre 29 e 10 dias. No início da fase adulta a $e_{x}$ dos parasitóides foi de 12,$0 ; 11,5 ; 10,6 ; 10,2$; 8,3 e 5,4 dias para as temperaturas de $18,21,24,27,30$ e $33^{\circ} \mathrm{C}$, respectivamente (Figura 2). Desta forma, pode-se observar que à medida que temperatura se eleva a esperança de vida deste parasitóide reduz.

\section{DISCUSSÃo}

O período ovo-adulto de $T$. pretiosum linhagem Tspd em ovos de $T$. ni variou entre as temperaturas estudadas apresentando uma relação inversa ao aumento da temperatura (Figura 1A). Para o parasitóide se desenvolver de ovo a adulto a $33^{\circ} \mathrm{C}$ foi necessário tempo 3,7 vezes menor que a $18^{\circ} \mathrm{C}$ (Figura $1 \mathrm{~B}$ ). Da mesma forma, a longevidade das fêmeas adultas apresentou a mesma tendência, decrescendo com a elevação da temperatura (Figura 1B). A $33^{\circ} \mathrm{C}$, a longevidade das fêmeas demonstrou uma redução de 2,2 e 1,9 vezes maior em relação às observadas a 18 e $24^{\circ} \mathrm{C}$ (Figura $1 \mathrm{~B}$ ). Esses resultados indicam a grande influência da temperatura sobre o desenvolvimento deste parasitóide.

A temperatura representa um dos principais mecanismos externos de regulação fisiológica dos seres vivos e, dentre os quais, os insetos são fortemente influenciados por este, vindo a sofrer bruscas alterações em seu desenvolvimento (CHOwn \& NiCHOLSON 2004; Speitht et al. 2008). Entretanto, a resposta fisiológica do parasitóide à variação de temperatura pode ser uma característica intrínseca, podendo variar entre espécies e, ou linhagens, bem como ser influenciada pelas características do hospedeiro em que se encontra (Pratissoli et al. 2008).

A influência negativa da temperatura sobre o período de desenvolvimento ovo-adulto tem sido relatada por vários autores para diferentes espécies de Trichogramma e hospedeiros (CALviN et al. 1984; Pratissoli \& Parra 2000; Pratissoli et al. 2006; Melo et al. 2007). FoERSTER \& FoERSTER (2009) ao estudarem cinco espécies de Trichogramma sobre Anticarsia gemmatalis Hübner (Lepidoptera: Noctuidae), relataram um decréscimo no período de desenvolvimento ovo-adulto com a elevação da temperatura, para todas as espécies estudadas. Além disso, esses autores verificaram ainda uma diferenciação entre as distintas espécies, sendo que a $18^{\circ} \mathrm{C}$ Trichogramma atopovirilia Oatman \& Platner apresentou a maior duração (19,0 dias) enquanto que as espécies T. pretiosum e Trichogramma rojasi Nagaraja \& Nagarkatti as menores (17,8 e 17,0 dias, respectivamente).

Apesar da percentagem de emergência não ter variado na faixa entre 18 e $33^{\circ} \mathrm{C}$, os resultados obtidos foram superiores aos encontrados por BoRBA et al. (2006). Esses autores relataram uma variação a $25^{\circ} \mathrm{C}$ de 61,7 a $68,3 \%$ em ovos de Bonagota cranaodes Meyrick (Lepidoptera: Totricidae) e de 85,4 a 89,6 \% em ovos de A. kuehniella ao trabalharem com T. pretiosum. Bueno et al. (2009), por sua vez, estudando T. pretiosum encontraram valores semelhantes entre A. gemmatalis e Pseudoplusia includens Walker (Lepidoptera: Noctuidae) sendo estes superiores a 90\%. Diante a isso, pode-se dizer que T. ni representa ser um hospedeiro em potencial para esta espécie de Trichogramma.

Com relação ao número de descendentes de T.pretiosum linhagem Tspd emergidos por ovo de $T$. ni, esse resultado demonstra que T. ni representa ser um bom hospedeiro para este parasitóide, pois T. pretiosum linhagem Tspd ovipositou mais de um ovo por ovo hospedeiro. Molina \& PARRA (2006) estudando 10 linhagens de $T$. pretiosum em Gymnandrosoma aurantianum Lima (Lepidoptera: Tortricidae) verificaram uma oscilação entre 1,4 a 1,8 indivíduos/ovo na temperatura de $25^{\circ} \mathrm{C}$, demonstrando que dentro da mesma espécie pode ocorrer diferenciação. Contudo, BuENo et al. (2009) relataram em seu estudo uma diferenciação significativa entre os hospedeiro $P$. includens e A. gemmatalis para este parâmetro em T. pretiosum. Segundo ÖzDER \& KARA (2010), o hospedeiro influencia diretamente o desenvolvimento dos parasitóides, visto que ele é a fonte de alimento e proteção para estes. Em função disso, o tamanho do hospedeiro não vai influenciar apenas o número de ovos depositados pela fêmea, mas também o tamanho do adulto de Trichogramma, o qual depende dos recursos nutricionais disponíveis para o desenvolvimento da larva (VINSON 1997).

Independente da razão de sexos não ter sofrido variação significativa com a variação de temperatura, os resultados encontrados estão dentro da faixa encontrada por outros autores (Perreira et al. 2007; Dias et al. 2008; Bueno et al. 2009; Pratissoli et al. 2010).

Resultados semelhantes para sobrevivência de $T$. pretiosum linhagem Tspd foram encontrados por LASHGARI et al. (2010) em
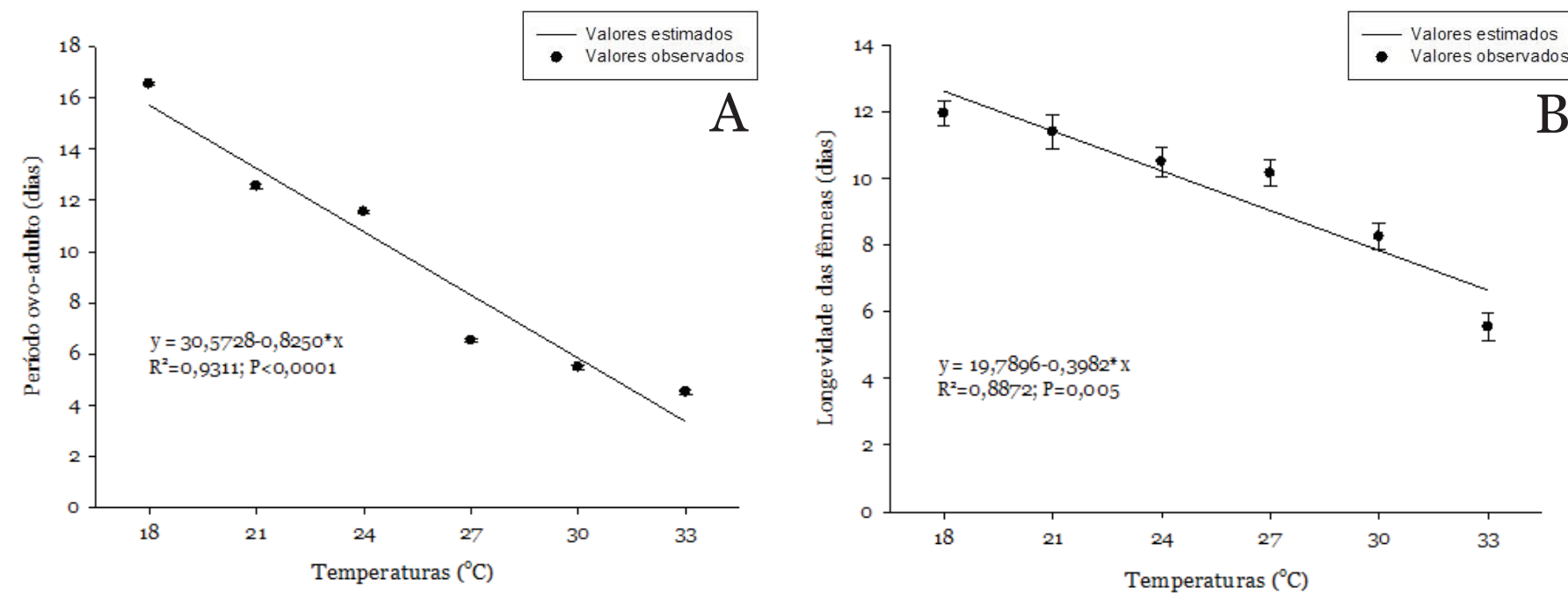

Figura 1. Parâmetros biológicos de Trichogramma pretiosum criado em ovos de Trichoplusia ni em diferentes temperaturas: período ovo-adulto (A), longevidade de fêmeas (B). 

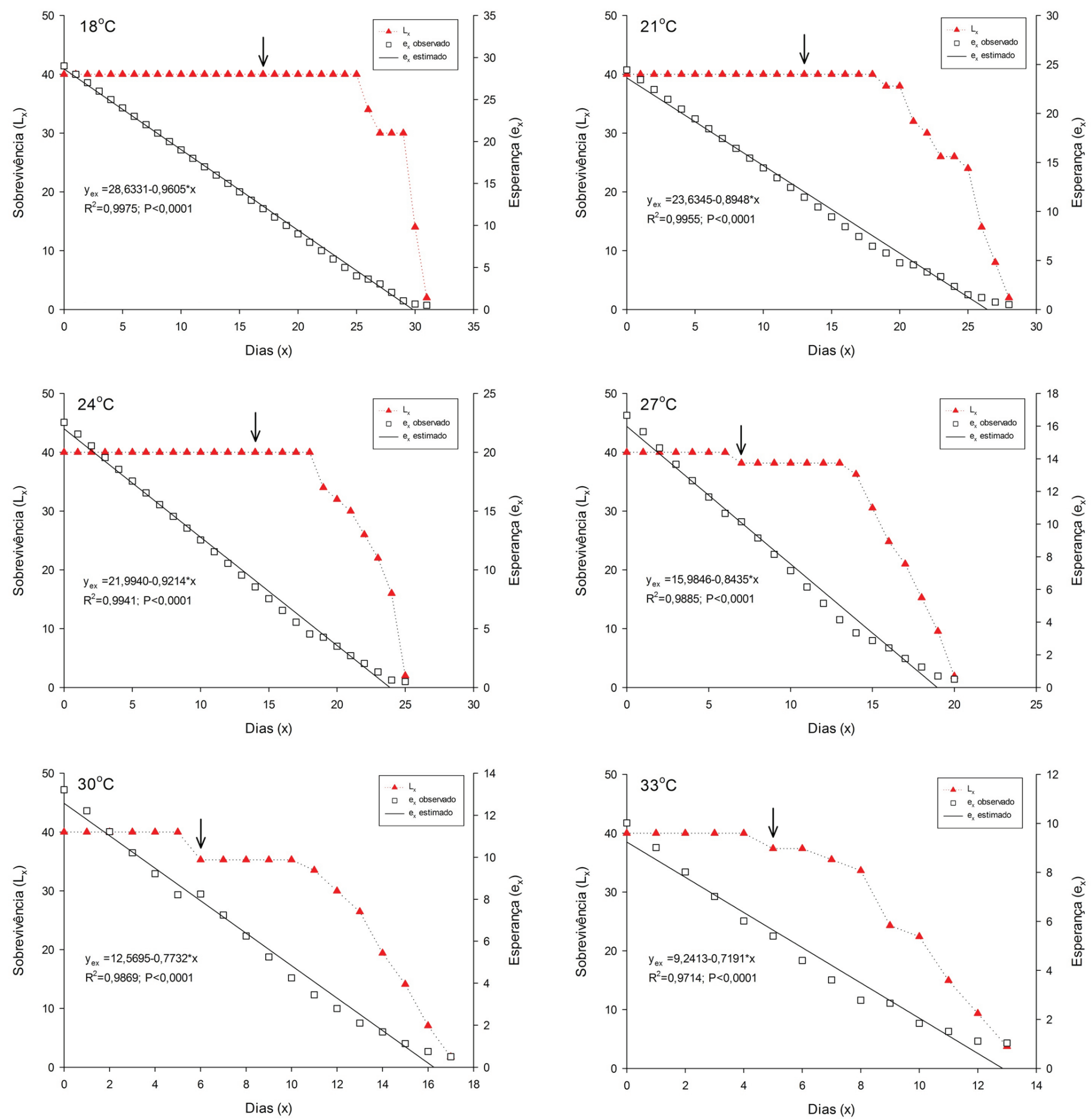

Figura 2. Número de sobrevivente (Lx) e esperança de vida (ex) de Trichogramma pretiosum em ovos de Trichoplusia ni em diferentes temperaturas. Seta $(\downarrow)$ indica o início da fase adulta.

sua pesquisa com Trichogramma brassicae Bezdenko. Esses autores verificaram uma sobrevivência de até 25 dias para $T$. brassicae nos hospedeiros A. kuehniella, Sitotroga cerealella Oliver (Lepidoptera: Gelechiidae) e Helicoverpa armigera Hübner (Lepidoptera: Noctuidae) a $25^{\circ} \mathrm{C}$. Além disso, eles observaram uma maior $d_{x}$ e uma $100 q_{x}$ de $T$. brassicae a partir do $13^{\circ}$ e $20^{\circ}$ dias de idade, respectivamente.

SAMARA et al. (2011), por sua vez, ao avaliarem cinco linhagens de Trichogramma aurosum Sugonjaev \& Sorokina em Cydia pomonella L. (Lepidoptera: Tortricidae), sob diferentes condicionamentos térmicos constantes, verificaram resultados semelhantes aos nossos para a mortalidade das fêmeas. Todavia, esses autores observaram valores de sobrevivência superiores ao desta pesquisa nas temperaturas 20,25 e $30^{\circ} \mathrm{C}$, sendo que a $20^{\circ} \mathrm{C}$ foram observados sobrevivências superiores a 16 dias. Da mesma forma, ÖZDER \& KARA (2010) relataram a mortalidade de 100\% dos adultos de Trichogramma cacoeciae Marchall, Trichogramma evanescens Westwood e T. brassicae a $30^{\circ} \mathrm{C}$ somente após o décimo dia de idade, quando criados em Cadra cautella Walker e Ephestia kuehniella Zeller (Lepidoptera: Pyralidade). A $20^{\circ} \mathrm{C}$ esses pesquisadores verificaram para as três espécies estudadas uma variação na sobrevivência de 25 a 28 dias entre os hospedeiros C. cautela e E. kuehniella, respectivamente.

A elevação da temperatura proporcionou uma redução da expectativa de vida dos parasitóides. Contudo, por mais que a temperatura venha a influenciar a esperança de vida dos parasitóide, a qualidade do hospedeiro também representa um fator crucial no seu desenvolvimento (VINSON 1997; PrATissoli et al. 2008). Este fato também foi observado por LASHGARI et al. (2010). Esses autores, avaliando T. brassicae em diferentes hospedeiros na temperatura de $25^{\circ} \mathrm{C}$, relataram uma esperança de vida para os adultos do parasitóide de 5,65; 9,45 e 4,95 dias em ovos de $H$. 
armigera, E. kuehniella e $S$. cerealella, respectivamente.

Desta forma, verificou-se que a temperatura exerce grande influência no desenvolvimento e sobrevivência de $T$. pretiosum em ovos de T. ni e que, T. ni constitui um bom hospedeiro para T. pretiosum linhagem Tspd com relação aos resultados obtidos para viabilidade e número de descendentes.

\section{REFERÊNCIAS}

Borba, R.S., M.S. Garcia, A. Kovaleski, A. Comioto \& R.L. Cardoso, 2006. Biologia e exigências térmicas de Trichogramma pretiosum Riley (Hymenoptera: Trichogrammatidae) sobre ovos de Bonagota cranaodes (Meyrick) (Lepidoptera: Tortricidae). Ciência Rural, 36: 1345-1352.

Bueno, R.C.O.F., J.R.P. Parra, A.F. Bueno \& M. Haddad, 2009. Desempenho de Tricogramatídeos como potenciais agentes de controle de Pseudoplusia includens Walker (Lepidoptera: Noctuidae). Neotropical Entomology, 38: 389-394.

Burkness, E.C. \& W.D. Hutchison, 2009. Implementing ReducedRisk Integrated Pest Management in Fresh-Market Cabbage: Influence of Sampling Parameters, and Validation of Binomial Sequential Sampling Plans for the Cabbage Looper (Lepidoptera: Noctuidae). Journal of Economic Entomology, 102: 1874-1883.

Calvin, D.D., M.C. Knapp, S.M. Welch, F.L. Poston \& R.J. Elzinga, 1984. Impact of environmental factors on Trichogramma pretiosum reared on southwestern corn borer eggs. Environmental Entomology, 13: 774-780.

Chown, S.L. \& S.W. Nicholson, 2004. Letal temperatura limits, p. 115-153. In: Chown S.L. \& S.W. Nicholson (Eds.). Insect Physiological Ecology: Mechanisms and Patterns, Oxford University Press, Oxford, 243p.

Dias, N.S., J.R.P. Parra \& T.C.C. Lima, 2008. Seleção de hospedeiro alternativo para três espécies de tricogramatídeos neotropicais. Pesquisa Agropecuária Brasileira, Brasília, 43: 1467-1473.

Foerster, M.R. \& L.A. Foerster, 2009. Effects of temperature on the immature development and emergence of five species of Trichogramma. BioControl, 54: 445-450.

Godin, C. \& G. Boivin, 1998. Lepidopterous pests of Brassica crops and their parasitoids in southwestern Quebec. Environmental Entomology, 27: 1157-1165.

Grecco, E.D., R.A. Polanczyk \& D. Pratissoli, 2010. Seleção e caracterização molecular de Bacillus thuringiensis Berliner com atividade tóxica para Trichoplusia ni Hübner (Lepidoptera: Noctuidae). Arquivos do Instituto Biológico, 77: 685-692.

Greene, G.L., N.C. Leppla \& W.A. Dickerson, 1976. Velvetbean caterpillar: rearing procedure and artificial medium. Journal of Economic Entomology, 69: 487-488.

Hassan, S.A. 1997. Seleção de espécies de Trichogramma para o uso em programas de controle biológico, p.183-205. In: Parra, J.R.P. \& R.AZucchi. (Eds.). Trichogramma e o Controle Biológico Aplicado. Piracicaba, FEALQ, 384p.

Iranipour, S., N. Vaez, G.N.Ghanbalani, R.A. Zakaria, \& M.M. Jafarloo, 2010. Effect of host change on demographic fitness of the parasitoid, Trichogramma brassicae. Journal of Insect Science, 10: 1-12.

Janmaat, A.F. \& J. Myers, 2003. Rapid evolution and the cost of resistance to Bacillus thuringiensis in greenhouse populations of cabbage loopers, Trichoplusia ni. Proceedings of the Royal Society of London, 270: 2263-2270.

Krebs, C.J. 1994. Ecology: The experimental analysis of distribution and abundance. New York, Harper \& Row, 801p.

Lashgari, A.A., A.A. Talebi, Y. Fathipour \& S. Farahani, 2010. Study on demographic parameters of Trichogramma brassicae (Bezdenko) (Hym., Trichogrammatidae) on three host species in laboratory conditions. Journal of Entomological Research, 2: $49-60$.
Melo, R. L., D. Pratissoli, R.A. Polanczyk, D.F. Melo, R. Barros \& A.M. Milanez, 2007. Biologia e Exigências Térmicas de Trichogrammaatopovirilia Oatman\&Platner(Hymenoptera: trichogrammatidae) em Ovos de Diaphania hyalinata L. (Lepidoptera: Pyralidae). Neotropical Entomology, 36: 431435.

Milanez, A.M., D. Pratissoli, R.A. Polanczyk, A.F. Bueno \& C.B.A. Tufik, 2009. Avaliação de Trichogramma spp. para o controle de Trichoplusia ni. Pesquisa Agropecuária Brasileira, 44: 1219-1224.

Molina, R.M.S. \& J.R.P. Parra, 2006. Seleção de linhagens de Trichogramma (Hymenoptera, Trichogrammatidae) e determinação do número de parasitóides a ser liberado para o controle de Gymnandrosoma aurantianum Lima (Lepidoptera, Tortricidae). Revista Brasileira de Entomologia, 50: 534-539.

Özder, N. \& G. Kara, 2010. Comparative biology and life tables of Trichogramma cacoeciae, T. brassicae and T. evanescens (Hymenoptera:Trichogrammatidae) with Ephestia kuehniella and Cadra cautella (Lepidoptera: Pyralidae) as hosts at three constant temperatures. Biocontrol Science and Technology, 20: $245-255$.

Parra, J.R.P., P.S.M. Botelho, B.S. Corrêa-Ferreira \& J.M.S. Bento, 2002. Controle biológico: terminologia, p.1-16. In: Bento, J.M.S. (Ed.). Controle biológico no Brasil: parasitóides e predadores. São Paulo, Manole, 626p.

Pereira, F.F., R. Barros, D. Pratissoli, C.L.T. Pereira, U.R. Vianna \& J.C. Zanuncio, 2007. Capacidade de parasitismo de Trichogramma exiguum Pinto \& Platner, 1978(Hymenoptera: Trichogrammatidae) em ovos de Plutella xylostella (L., 1758) (Lepidoptera: Plutellidae) em diferentes temperaturas. Ciência Rural, 37: 297-303.

Pratissoli, D. \& J.R.P. Parra, 200o. Desenvolvimento e exigências térmicas de Trichogramma pretiosum Riley, criados em duas traças do tomateiro. Pesquisa Agropecuária Brasileira, 35: 1281-1288.

Pratissoli, D., L.P. Dalvi, R.A. Polanczyk, G.S. Andrade, A.M. Holtz \& H.O. Nicoline, 2010. Características biológicas de Trichogramma exiguum em ovos de Anagasta kuehniella e Sitotroga cerealella. Idesia, 28: 39-42.

Pratissoli, D., M.J. Fornazier, A.M. Holtz, J.R. Gonçalves, A.B. Chioramital \& H.B.Zago, 2002. Ocorrência de Trichogramma pretiosum em áreas comerciais de tomate, no Espirito Santo, em regiões de diferentes altitudes. Horticultura Brasileira, 21: 73-76.

Pratissoli, D., R.A. Polanczyk, A.M. Holtz, L.P. Dalvi, A.F. Silva \& L.N. Silva, 2008. Selection of Trichogramma species for controlling the Diamondback moth. Horticultura Brasileira, 26: $259^{-261 .}$

Pratissoli, D., E.F. Reis, H.B. Zago, P.L. Pastori \& T. Tamanhoni, 2006. Biologia e exigências térmicas de cinco linhagens de Trichogramma pretiosum Riley (Hymenoptera: Trichogrammatidae) criadas em ovos de Tuta absoluta (Meyrick) (Lepidoptera: Gelechiidae). Ciência Rural, 36: 1671-1677.

Samara, R., J.C. Monje, C.P.W. Zebitz \& T. Qubbaj, 2011. Comparative biology and life tables of Trichogramma aurosum on Cydia pomonella at constant temperatures. Phytoparasitica, 39: 109-119.

Silveira Neto, S., O. Nakano, D. Barbin \& N. A. V. Nova, 1976. Manual de ecologia dos insetos. São Paulo, Agronômica Ceres, 419p.

Speight, M.R., M.D. Hunter \& A.D. Watt, 2008. Ecology of insects: concepts and applications. Oxford: Wiley-Blackwell, $628 \mathrm{p}$.

Vinson, S. B., 1997. Comportamento de seleção hospedeira de parasitóides de ovos, com ênfase na família Trichogrammatidae, p. 67-120. In: Parra, J.R.P. \& R.A. Zucchi (Eds.). Trichogramma e o Controle Biológico Aplicado. Piracicaba, FEALQ, 384p. 
Recebido em: 12/o1/2012

Aceito em: 14/o6/2012

Como citar este artigo:

Carvalho, J.R., D. Pratissoli, I.P.S. Quadros, J.P.P. Paes, I.S. Kaiser, H.S. Rodrigues, A.C.Túller \& V. Zuim, 2012. Tabela de Esperança de Vida de

Trichogramma pretiosum Riley em Ovos de Trichoplusia ni Hübner em Diferentes Condicionamentos Térmicos. EntomoBrasilis, 5(3): 173-178. Acessível em: http://www.periodico.ebras.bio.br/ojs/index.php/ebras/article/view/222

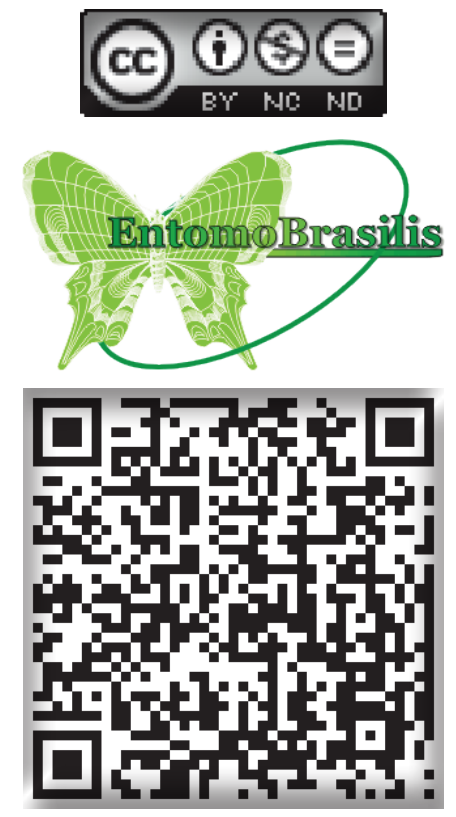

